KUNS-1803

hep-ph/0209088

\title{
Higgs Doublets as Pseudo Nambu-Goldstone Bosons in Supersymmetric $\boldsymbol{E}_{6}$ Unification
}

\author{
Masako BANDO ${ }^{1, *)}$ and Taichiro KUGO**) \\ ${ }^{1}$ Physics Division, Aichi University, Aichi 470-0296, Japan \\ Department of Physics, Kyoto University, Kyoto 606-8502, Japan
}

\begin{abstract}
The idea to have Higgs doublets as pseudo Nambu-Goldstone (PsNG) multiplets is examined in the framework of supersymmetric $E_{6}$ unified theory. We show that extra PsNG multiplets other than the expected Higgs doublets necessarily appear in the $E_{6}$ case. If we demand that the extra PsNG multiplets neither disturb the gauge coupling unification nor make the color gauge coupling diverge before unification occurs, only possibility for the extra PsNG is $\mathbf{1 0}+\overline{\mathbf{1 0}}$ of $S U(5)$. This is realized when the symmetry breaking $E_{6} \rightarrow S O(10)$ occurs in the $\phi(\mathbf{2 7})+\phi(\overline{\mathbf{2 7}})$ sector while $E_{6} \rightarrow$ $S U(4)_{C} \times S U(2)_{L} \times U(1) \times U(1)$ in the $\Sigma(\mathbf{7 8})$ sector. The existence of $\mathbf{1 0}+\overline{\mathbf{1 0}}$ multiplets with mass around $1 \mathrm{TeV}$ is therefore a prediction of this $E_{6}$ PsNG scenario. Implication of their existence on the proton decay is also discussed.
\end{abstract}

\footnotetext{
*) E-mail: bando@aichi-u.ac.jp

**) E-mail: kugo@gauge.scphys.kyoto-u.ac.jp
} 
There are many attractive features of grand unified theories (GUT), such as gauge unification, miraculous anomaly cancellation within a family, charge quantization, etc.. In the present form of GUT, however, there are also many unsolved problems. One of the most serious difficulties would be the so-called hierarchy problem; we need extremely light Higgs doublets which are responsible for breaking electroweak symmetry, and their masses should be kept light against radiative corrections. The most attractive way to protect against such radiative correction is to introduce supersymmetry (SUSY), which is not yet confirmed by experiments; no superpartner has been observed. Another aspect of the hierarchy problem is the so-called doublet-triplet (DT) splitting problem. It is not yet made clear how we can naturally split only $S U(2)_{L}$ doublets from their GUT partner color triplet states. There have been many attempts to solve this problem; ${ }^{1)-4)}$ missing partner mechanism, sliding singlet mechanizm, Dimopoulos-Wilczek mechanism, etc.. Among various approaches we concentrate in this paper our attention on the simplest idea which has long been investigated, namely the idea that Higgs doublets are realized as pseudo Nambu Goldstone (PsNG) bosons. 5) - 10) Actually in supersymmetric grand unified scenario, once PsNG multiplets appear, they are kept massless so far as SUSY remains unbroken because of nonrenormalization theorem. Usually light PsNG multiplets are not welcome since their additional contributions to RGE harm the gauge unification. However if we can identify them as the usual Higgs doublets, we can make active use of such property of PsNG modes in explaining the light Higgs doublets.

This letter aims to examine this idea of PsNG in supersymmetric $E_{6}$ unified theories. The idea of PsNG has been first proposed by Inoue, Kakuto and Takano in $1986^{5)}$ adopting a global $S U(6)$ whose subgroup $S U(5)$ is gauged. Later it was made more realistic by Barbieri, Dvali and Moretti $^{8)}$ by taking local $S U(6)$ symmetry and utilizing two Higgs sectors possessing no cross couplings. Dvali and Pokorski ${ }^{10)}$ pointed out that the anomalous $U(1)_{X}$ symmetry can play a role in making two Higgs sectors separated from each other in the superpotential term. An extension to $E_{6}$ gauge symmetry was considered in Ref. 9) with a negative result.

Consider a supersymmetric grand unified theory based on a gauge group $G$. Suppose that the theory possesses two 'Higgs scalar fields', $\phi$ and $\Sigma$, each of which need not be of irreducible representation of $G$ so that they each may actually stand for a set of fields. The point is that we assume that they have no direct cross couplings in the superpotential,

$$
W=W_{1}(\phi)+W_{2}(\Sigma)
$$

so that the superpotential has an enhanced symmetry $G_{\phi} \times G_{\Sigma}$, invariance under separate rotations of $\phi$ - and $\Sigma$-sectors. In principle $G_{\phi}$ and $G_{\Sigma}$ can be (accidentally) larger than the 
gauge group $G$, but here we assume that both are $G ; G_{\phi}=G_{\Sigma}=G$. Suppose that $\phi$ and $\Sigma$ develop their vacuum expectation values (VEVs) $\langle\phi\rangle$ and $\langle\Sigma\rangle$ and the symmetries are broken into

$$
\begin{array}{lllll}
G_{\phi}=G & \rightarrow & H_{\phi} & \text { by } & \langle\phi\rangle, \\
G_{\Sigma}=G & \rightarrow & H_{\Sigma} & \text { by } & \langle\Sigma\rangle .
\end{array}
$$

Then, the Nambu-Goldstone (NG) multiplets corresponding to the cosets $G / H_{\phi}$ and $G / H_{\Sigma}$ appear from the $\phi$ and $\Sigma$ sectors, respectively. But the actual symmetry of the full system is only $G$ and it is broken to the intersection subgroup $H_{\phi} \cap H_{\Sigma}$, so that the true NG multiplets are only those of $G /\left(H_{\phi} \cap H_{\Sigma}\right)$. The other multiplets not contained in $G /\left(H_{\phi} \cap H_{\Sigma}\right)$ are therefore all pseudo Nambu-Goldstone (PsNG) multiplets, whose number is counted as *)

$$
\begin{aligned}
\text { \# of PsNG multiplets } & =\operatorname{dim}\left[G / H_{\phi}\right]+\operatorname{dim}\left[G / H_{\Sigma}\right]-\operatorname{dim}\left[G /\left(H_{\phi} \cap H_{\Sigma}\right)\right] \\
& =\operatorname{dim} G+\operatorname{dim}\left[H_{\phi} \cap H_{\Sigma}\right]-\operatorname{dim} H_{\phi}-\operatorname{dim} H_{\Sigma} .
\end{aligned}
$$

Before entering the main subjects, we here comment on the the fact that exactly the same contents of PsNG multiplets also appear under a slightly different setup which was originally considered by K. Inoue and A. Kakuto and H. Takano. The setup they considered is as follows: the gauge symmetry $G_{\text {local }}$ of the system is $H_{\Sigma}$, and the superpotential of the Higgs fields $\phi$ of the system possesses a global symmetry $G_{\text {global }}=G$ larger than the required local symmetry $G_{\text {local }}$ and $\phi$ develops a VEV which retains only a symmetry $H_{\phi}$. We call this setup 'global $G$ setup' while the above one our 'local $G$ setup'. Note that we can exchange $H_{\phi}$ and $H_{\Sigma}$ in this global $G$ setup since our local $G$ setup is symmetric under the exchange $H_{\phi} \leftrightarrow H_{\Sigma}$.

The reason why the same contents of PsNG multiplets appear in both setups is as follows: Suppose that the VEV $\langle\Sigma\rangle$ is much larger than the VEV $\langle\phi\rangle$ in our local $G$ setup. Then we can consider an effective theory at the energy scale lower than $\langle\Sigma\rangle$ but higher than $\langle\phi\rangle$. There the original local symmetry $G$ is already spontaneously broken to $H_{\Sigma}$ and the associated NG multiplets of $G / H_{\Sigma}$ are all absorbed in the $G$-gauge multiplet. The rest components of $\Sigma$ become massive of order $\langle\Sigma\rangle$ and decouple. Therefore the system at this stage is just the same as that of the global $G$ setup with Higgs fields $\phi$. Indeed the superpotential of $\phi$ retains the symmetry $G$ as a global symmetry while the local gauge symmetry of the system is only $H_{\Sigma}$. This finishes the proof. In this proof we have assumed $\langle\Sigma\rangle \gg\langle\phi\rangle$. But the number counting of broken generators is clearly independent of such an ordering, so the proof is generally valid.

\footnotetext{
*) This counting corresponds to the so-called maximum realization case. ${ }^{11)}$
} 
First let us use the following notation ${ }^{10)}$ for the generated NG multiplets according to the representations under the standard theory gauge symmetry $G_{S}=S U(3)_{C} \times S U(2)_{L} \times U(1)_{Y}$ :

$$
\begin{aligned}
\hat{Q}_{Y} & =(3,2)_{Y}+(\overline{3}, 2)_{-Y}, \\
\hat{T}_{Y} & =(3,1)_{Y}+(\overline{3}, 1)_{-Y}, \\
\hat{D}_{Y} & =(1,2)_{Y}+(1,2)_{-Y}=\hat{D}_{-Y}, \\
S_{Y} & =(1,1)_{Y} .
\end{aligned}
$$

where the two numbers in each bracket stand for the dimensions of the representations of $S U(3)_{C}$ and $S U(2)_{L}$, and the attached suffix for the value of the hypercharge $Y$. We will also use notation like $\hat{Q}$ when we do not specify the hypercharge value.

First of all let us find the representations of the true NG multiplets which appear when the group $E_{6}$ breaks down to the standard theory gauge group $G_{S}=S U(3)_{C} \times S U(2)_{L} \times U(1)_{Y}$. The adjoint representation $\mathbf{7 8}$ of $E_{6}$ is decomposed into irreducible representations of the subgroup $S O(10)$ as

$$
78=45+1+16+\overline{16}
$$

and the $S O(10)$ adjoint $\mathbf{4 5}$ and the spinor $\mathbf{1 6}$ are further decomposed into $S U(5)$ representations as

$$
\begin{aligned}
& 45=24+1+10+\overline{10} \\
& 16=10+\overline{5}+1
\end{aligned}
$$

As is well-known, these $S U(5)$ representations 24, 10 and $\overline{\mathbf{5}}$ are decomposed under the standard theory gauge symmetry $G_{S}$ as ${ }^{12)}$

$$
\begin{aligned}
\mathbf{2 4} & =(\mathbf{8}, \mathbf{1})_{0}+(\mathbf{1}, \mathbf{3})_{0}+(\mathbf{1}, \mathbf{1})_{0}+(\mathbf{3}, \mathbf{2})_{-5 / 6}+(\overline{\mathbf{3}}, \mathbf{2})_{5 / 6}, \\
\mathbf{1 0} & =(\mathbf{3}, \mathbf{2})_{1 / 6}+(\overline{\mathbf{3}}, \mathbf{1})_{-2 / 3}+(\mathbf{1}, \mathbf{1})_{-1}, \\
\overline{\mathbf{5}} & =(\overline{\mathbf{3}}, \mathbf{1})_{1 / 3}+(\mathbf{1}, \mathbf{2})_{-1 / 2} .
\end{aligned}
$$

Therefore, when $E_{6}$ breaks down to $S O(10)$, the NG multiplets appearing are given by

$$
\begin{aligned}
E_{6} \rightarrow S O(10): \mathbf{1 6} & +\overline{\mathbf{1 6}}+\mathbf{1}=(\mathbf{1 0}+\overline{\mathbf{1 0}})+(\overline{\mathbf{5}}+\mathbf{5})+3 \times \mathbf{1}, \\
& =\left(\hat{Q}_{1 / 6}+\hat{T}_{2 / 3}+S_{1}+S_{-1}\right)+\left(\hat{T}_{-1 / 3}+\hat{D}_{1 / 2}\right)+3 S_{0},
\end{aligned}
$$

and, when $S O(10)$ further breaks down to $S U(5)$ and then to the standard theory gauge group $G_{S}$, the appearing NG multiplets are

$$
\begin{gathered}
S O(10) \rightarrow S U(5): \mathbf{1 0}+\overline{\mathbf{1 0}}+\mathbf{1}=\left(\hat{Q}_{1 / 6}+\hat{T}_{2 / 3}+S_{1}+S_{-1}\right)+S_{0}, \\
S U(5) \rightarrow G_{S}:(\mathbf{3}, \mathbf{2})_{-5 / 6}+(\overline{\mathbf{3}}, \mathbf{2})_{5 / 6}=\hat{Q}_{-5 / 6} .
\end{gathered}
$$


The net NG multiplets appearing when $E_{6}$ breaks down to the standard theory gauge group $G_{S}$ is thus found to be

$$
2\left(\hat{Q}_{1 / 6}+\hat{T}_{2 / 3}+S_{1}+S_{-1}\right)+\hat{Q}_{-5 / 6}+\left(\hat{T}_{-1 / 3}+\hat{D}_{1 / 2}\right)+4 S_{0}
$$

Next, as another breaking pattern, we consider the breaking of $E_{6}$ into its maximal subgroup $S U(6) \times S U(2)$. The adjoint $\mathbf{7 8}$ decomposes under $S U(6) \times S U(2)$ as

$$
78=(1,3)+(35,1)+(20,2)
$$

where the $S U(6) \mathbf{2 0}$ of broken generator $(\mathbf{2 0 , 2})$ is further decomposed under the subgroup $S U(4) \times S U(2) \subset S U(6)$ into

$$
\mathbf{2 0}=(\mathbf{4}, \mathbf{1})+(\overline{4}, \mathbf{1})+(\mathbf{6}, \mathbf{2}) \quad(\leftarrow \theta=\mathrm{g}+\mathrm{\theta}+\mathrm{\theta}) .
$$

(The undotted and dotted boxes in the Young tableau on the right-hand side stand for the indices of $S U(4)$ and $S U(2)$ of the subgroup $S U(4) \times S U(2) \subset S U(6)$, respectively.) If the first factor group $S U(6)$ contains both $S U(3)_{C}$ and $S U(2)_{L}$ of the standard theory gauge group $G_{S}$, in which case $S U(6)$ is denoted as $S U(6)_{C, L}$, the NG multiplets associate with the breaking $E_{6} \rightarrow S U(6)_{C, L} \times S U(2)$ are given by

$$
2 \times \mathbf{2 0}=2 \times\{((\mathbf{3}, \mathbf{1})+(\overline{\mathbf{3}}, \mathbf{1}))+2(\mathbf{1}, \mathbf{1})+((\mathbf{3}, \mathbf{2})+(\overline{\mathbf{3}}, \mathbf{2}))\}=2 \hat{Q}+2 \hat{T}+4 S .
$$

Here we have not specified the hypercharge values since there are various possibilities how $U(1)_{Y}$ generators are embedded in the unbroken subgroup. On the other hand, if $S U(3)_{C}$ is contained in the first $S U(6)$ while $S U(2)_{L}$ in the second $S U(2)$, i.e., $E_{6}$ breaks down to $S U(6)_{C} \times S U(2)_{L}$, then the resultant NG multiplets are given by

$$
(\mathbf{2 0}, \mathbf{2})=3 \times(\mathbf{3}, \mathbf{2})+3 \times(\overline{\mathbf{3}}, \mathbf{2})+2 \times(\mathbf{1}, \mathbf{2})=3 \hat{Q}+\hat{D} .
$$

Now let us consider the breaking patterns of $E_{6}$ into subgroups $H$ where $H$ contains the standard theory gauge group $G_{S}=S U(3)_{C} \times S U(2)_{L} \times U(1)_{Y}$. In order to exhaust all the possibilities of the breaking patterns $E_{6} \rightarrow H$ in a systematic way, we first classify the cases by identifying only the part $\tilde{H}$ of the subgroup $H$ containing the $S U(3)_{C}$ and $S U(2)_{L}$ groups of $G_{S}$. That is, we do not identify how the hypercharge $U(1)_{Y}$ is contained in the full $H$ and neglect the part (factor group) of $H$ which contains neither $S U(3)_{C}$ nor $S U(2)_{L}$. For instance, the choices of $H=S U(4)_{C} \times S U(2)_{L} \times S U(2) \times U(1)$ and $H=S U(4)_{C} \times S U(2)_{L} \times[U(1)]^{k}$ $(k=0,1,2)$ are all classified into the case $\tilde{H}=S U(4)_{C} \times S U(2)_{L}$. The suffices $C$ and $L$ attached to the group name always mean that the $S U(3)_{C}$ and $S U(2)_{L}$ groups of $G_{S}$ are contained in that group, as we have defined in the above. This greatly simplify the task. 
We classify the possibilities of the choice of $\tilde{H}$ according to its rank. The maximal regular subgroups of $E_{6}$ are $S U(6) \times S U(2), S O(10) \times U(1)$ and $[S U(3)]^{3}$. However, since we only specify the factor groups that contain $S U(3)_{C}$ and $S U(2)_{L}$, then only possibilities of $\tilde{H}$ are clearly $S U(6)_{C} \times S U(2)_{L}$ and $S U(6)_{C, L}$ for the first $S U(6) \times S U(2), S O(10)_{C, L}$ for the second $S O(10) \times U(1)$, and $S U(3)_{C} \times S U(3)_{L}$ for the third $[S U(3)]^{3}$. Lower rank cases of $\tilde{H}$ can be found by considering further breaking of these cases. In this way we find all the possibilities for $\tilde{H} \supset G_{S}$ and tabulate them in Table. I. There we also list the representations of the NG multiplets under $\tilde{H}$ appearing in each breaking $E_{6} \rightarrow \tilde{H}$.

Table I. Possible choices for $\tilde{H} \supset G_{S}$ and NG fields for the breaking $E_{6} \rightarrow \tilde{H}$. The columns $\hat{Q}, \hat{T}$ and $\hat{D}$ denote the numbers of times those representations of NG multiplets appear in $E_{6} / \tilde{H}$. $S U(3)_{C} \times S U(2)_{L}$ singlets are neglected.

\begin{tabular}{|c|c|c|c|c|c|c|}
\hline \hline rank & Name & $\tilde{H}$ & repr. under $\tilde{H}$ of the coset $E_{6} / \tilde{H}$ & $\hat{Q}$ & $\hat{T}$ & $\hat{D}$ \\
\hline \hline 6 & $\mathrm{E}$ & $S U(6)_{C} \times S U(2)_{L}$ & $(\mathbf{2 0}, \mathbf{2})$ & 3 & 0 & 1 \\
\hline \hline 5 & $\mathrm{~A}$ & $S O(10)_{C, L}$ & $\mathbf{1 6}+\overline{\mathbf{1 6}}$ & 1 & 2 & 1 \\
\hline & $\mathrm{B}$ & $S U(5)_{C} \times S U(2)_{L}$ & $(\mathbf{1 0}, \mathbf{2})+(\overline{\mathbf{1 0}}, \mathbf{2})+(\mathbf{5}, \mathbf{1})+(\overline{\mathbf{5}}, \mathbf{1})$ & 3 & 1 & 1 \\
\hline & & $S U(6)_{C, L}$ & $2 \times \mathbf{2 0}$ & 2 & 2 & 0 \\
\hline \hline 4 & $\mathrm{C}$ & $S U(5)_{C, L}$ & $2 \times(\mathbf{1 0}+\overline{\mathbf{1 0}})+\mathbf{5}+\overline{\mathbf{5}}$ & 2 & 3 & 1 \\
\hline & $\mathrm{D}$ & $S U(4)_{C} \times S U(2)_{L}$ & $2 \times((\mathbf{6}, \mathbf{2})+(\mathbf{4}, \mathbf{1})+(\overline{\mathbf{4}}, \mathbf{1}))+(\mathbf{4}, \mathbf{2})+(\overline{\mathbf{4}}, \mathbf{2})$ & 3 & 2 & 1 \\
\hline & & $S U(3)_{C} \times S U(3)_{L}$ & $3 \times(\mathbf{3}, \mathbf{3})+3 \times(\overline{\mathbf{3}}, \overline{\mathbf{3}})$ & 3 & 3 & 0 \\
\hline \hline 3 & final & $S U(3)_{C} \times S U(2)_{L}$ & $3 \times(\mathbf{3}, \mathbf{2}+\mathbf{1})+3 \times(\overline{\mathbf{3}}, \mathbf{2}+\mathbf{1})+2 \times(\mathbf{1}, \mathbf{2})$ & 3 & 3 & 1 \\
\hline \hline
\end{tabular}

Since we specify how $\tilde{H}$ contains $S U(3)_{C}$ and $S U(2)_{L}$, we can count the numbers of appearing NG multiplets of representations $\hat{Q}, \hat{T}$ and $\hat{D}$, which are also shown in Table. I. We can not count the numbers of $S U(3)_{C} \times S U(2)_{L}$-singlet NG multiplets nor the hypercharges of the $S U(3)_{C} \times S U(2)_{L}$ non-singlet NG multiplets. They can be specified later in concrete cases after narrowing down the possibilities.

Now with Table. I, we can find all the possible choices of $\tilde{H}_{\phi}$ and $\tilde{H}_{\Sigma}$. The conditions which should be satisfied are: i) an $S U(2)_{L}$ doublet $\hat{D}$ appear as a PsNG multiplet, and ii) other PsNG multiplets, if exist, should fall into an $S U(5)_{\mathrm{GG}}$ multiplet so as not to disturb the gauge coupling unification.

From Table. I, we see that at most only one $\hat{D}$ NG multiplet can appear for any choices of $\tilde{H}$ and one $\hat{D}$ appears as a true NG multiplet in the $E_{6} \rightarrow G_{S}$ breakdown. In order to satisfy the condition i), therefore, we must have one $\hat{D}$ NG multiplet for each of breakings 
$E_{6} \rightarrow H_{\phi}$ and $E_{6} \rightarrow H_{\Sigma}$, and so the candidates for $\tilde{H}_{\phi}$ and $\tilde{H}_{\Sigma}$ are restricted to the cases A, B, C, D and E.

For any choice of a pair $\left(H_{\phi}, H_{\Sigma}\right)$ from A, B, C, D and E, we immediately see that extra PsNG multiplets appear other than the desired $\hat{D}$ in this $E_{6}$ case. Note that the sum of the numbers of appearing $\hat{Q}$ and $\hat{T}$ in the pair should be larger than or equal to three for both $\hat{Q}$ and $\hat{T}$ since the true NG multiplets are $3 \hat{Q}+3 \hat{T}+\hat{D}$. If the sum is less than 3 for either $\hat{Q}$ or $\hat{T}$, it implies that the intersection $H_{\phi} \cap H_{\Sigma}$ is larger than $G_{S}$ in contradiction to the assumption. Since no extra $\hat{D}$ other than the two (a true NG and a PsNG) multiplets appears, the only possibility for the $S U(5)$ multiplet into which other PsNG multiplets could fall is $\mathbf{1 0}+\overline{\mathbf{1 0}} \supset \hat{Q}+\hat{T}$, which contains no $\hat{D}$ and equal numbers of $\hat{Q}$ and $\hat{T}$. Therefore the sums of the numbers of appearing $\hat{Q}$ and $\hat{T}$ should be equal in order to satisfy the condition ii).

It is immediate to see that the only possible choices of such a pair satisfying this condition are $(\mathrm{A}, \mathrm{D})$ and $(\mathrm{C}, \mathrm{D})$. The former choice $(\mathrm{A}, \mathrm{D})$ yields $4 \hat{Q}+4 \hat{T}+2 \hat{D}$ so that it gives a $\mathbf{1 0}+\overline{\mathbf{1 0}}$ extra PsNG multiplets, while the latter case (C,D) gives $5 \hat{Q}+5 \hat{T}+2 \hat{D}$ containing two pairs of $\mathbf{1 0}+\overline{\mathbf{1 0}}$ extra PsNG multiplets. However we can see that the presence of $2 \hat{Q}+2 \hat{T}$ PsNG multiplets makes the $S U(3)_{C}$ gauge interaction asymptotically non-free and the coupling constant becomes infinity before reaching the unification scale. Indeed, we have the formula for the running coupling $\alpha=g^{2} / 4 \pi$ at one loop,

$$
\begin{aligned}
\frac{1}{\alpha(\mu)} & =\frac{1}{\alpha(M)}+\frac{b}{2 \pi} \ln \left(\frac{M}{\mu}\right), \\
b & =-\frac{9}{3} T(\operatorname{adj})+\sum_{R} N_{R} T(R) \quad \operatorname{tr}\left(T_{R}^{a} T_{R}^{b}\right)=T(R) \delta_{a b}
\end{aligned}
$$

where $N_{R}$ is the number of chiral multiplets of representation $R$, and the quadratic Casimir $T(\operatorname{adj}) \equiv C_{2}(G)$ is $N$ for $G=S U(N)$ and $T(\square)=1 / 2$ for the fundamental representation $\square$ and $T(\boxminus)=(N-2) / 2$ for the representation $\boxminus$. For $S U(3)_{C}$ gauge coupling and for three generations ( $6 \mathbf{3}+\overline{\mathbf{3}}$ chiral multiplets) plus two $\mathbf{1 0}+\overline{\mathbf{1 0}}$ PsNG multiplets $(2 \times(2+1)=6$ $3+\overline{3}$ chiral multiplets $)$, we have $b=-9+(6+6)(1 / 2+1 / 2)=3>0$, which makes $\alpha_{s}(\mu)$ diverge at around $\mu=6 \times 10^{9} \mathrm{GeV}$. We thus see that the only possibility is the choice (A,D). It is interesting that the presence of $\hat{Q}+\hat{T}$ in this case just makes the $\beta$ function of $S U(3)_{C}$ gauge coupling vanish at one-loop; $b=-9+(6+3)(1 / 2+1 / 2)=0$.

We thus have seen that the breaking pattern choice $(A, D)$ is the only possibility. However, this is only a necessary condition. It is quite non-trivial whether there is actually a concrete model of breaking pattern (A,D) which also satisfies the $U(1)_{Y}$ quantum number requirements, which we have not examined above.

It is sufficient to find a model that satisfies all the requirements. We consider a model 
in which $E_{6}$ is spontaneously broken to $S O(10)_{C, L}$ by fundamental and anti-fundamental repr. Higgs fields $\phi(\mathbf{2 7})$ and $\phi(\overline{\mathbf{2 7}})$, while it is broken down to $S U(4)_{C} \times S U(2)_{L} \times U(1)_{A} \times$ $U(1)_{B}$ by an adjoint Higgs $\Sigma(\mathbf{7 8})$ :

$$
\begin{array}{ll}
\text { A : } & E_{6} \rightarrow H_{\phi}=S O(10)_{C, L} \quad \text { by } \quad \phi(\mathbf{2 7}) \text { and } \phi(\overline{\mathbf{2 7}}), \\
\mathrm{D}: & E_{6} \rightarrow H_{\Sigma}=S U(4)_{C} \times S U(2)_{L} \times U(1)_{A} \times U(1)_{B} \quad \text { by } \quad \Sigma(\mathbf{7 8}) .
\end{array}
$$

(It should be noted that the breaking by adjoint $\Sigma$ cannot lower the rank of $H_{\Sigma}$ than that of $E_{6}$.) We shall specify these $S O(10)_{C, L}, S U(4)_{C}$ and $U(1)_{A} \times U(1)_{B}$ in more detail below by identifying which components of $\phi(\mathbf{2 7})$ and $\Sigma(\mathbf{7 8})$ acquire the VEVs. The requirements is that the intersection $H_{\phi} \cap H_{\Sigma}$ should be the standard model group $G_{S}$.

For that purpose, it is convenient to name all the twenty seven components of the fundamental representation $\phi(\mathbf{2 7}) . \mathbf{2 7}$ is decomposed as $\mathbf{2 7}=\mathbf{1 6}+\mathbf{1 0}+\mathbf{1}$ under GeorgiFritsch-Minkowski's $S O(10)_{\text {GFM }} \subset E_{6}$. Decomposing them further under Georgi-Glashow's $S U(5)_{\mathrm{GG}} \subset S O(10)$, we name the 27 components as follows: ${ }^{13)}$

$$
\begin{aligned}
& 16=\frac{10}{\left[u^{c i},\left(\begin{array}{l}
u_{i} \\
d_{i}
\end{array}\right), e^{c}\right]}+\frac{5^{*}}{} \quad+\quad \begin{array}{l}
\left(d^{c i}, e,-\nu\right) \\
\end{array} \\
& \mathbf{1 0}=\begin{array}{c}
\mathbf{5} \\
\left(D_{i}, E^{c},-N^{c}\right)
\end{array} \quad+\begin{array}{c}
\mathbf{5}^{*}, \\
\left(D^{c i}, E,-N\right)
\end{array}
\end{aligned}
$$

The simplest scenario for the breaking A is realized by the VEV of the $S O(10)$-singlet component $S$ of $\phi(\mathbf{2 7})$ :

$$
\langle\phi(\mathbf{1})=S(\phi)\rangle=v_{\phi}
$$

In this case the unbroken subgroup $H_{\phi}$ is Georgi-Fritsch-Minkowski's $S O(10)_{\text {GFM }}$ which contains Pati-Salam $S U(4)_{\mathrm{PS}} \simeq S O(6)$ and $S U(2)_{L} \times S U(2)_{R} \simeq S O(4)$ as its subgroup. But the choice of $S O(10)$ in $E_{6}$ even with a constraint $S O(10) \supset S U(5)_{\mathrm{GG}}$ is not unique at all but has a freedom of an $S U(2)$ rotation. Indeed as pointed out in Ref. 12), there is a maximal subgroup $S U(6) \times S U(2)_{E}$ in $E_{6}$, where $S U(6) \supset S U(5)_{\mathrm{GG}}$ and the $(5+1) \times 2$ components in 27

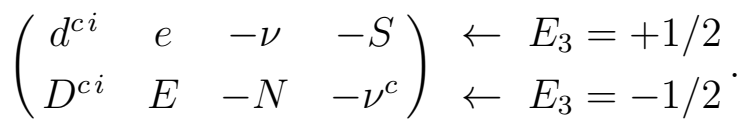

give an $S U(2)_{E}$ doublet of $S U(6)$ 6-plets. That is, the two $\mathbf{5}^{*}$-plets and two singlets 1 of $S U(5)_{\mathrm{GG}}$ in Eq. (21) are rotated into each other under the $S U(2)_{E}$. Since the generators of $S U(2)_{E}$ are orthogonal to those of $S U(5)_{\mathrm{GG}}$, the $S U(2)_{E}$-rotated $S O(10)$ from $S O(10)_{\mathrm{GFM}}$ with any angle $\boldsymbol{\theta}^{12)}$

$$
S O(10)_{\boldsymbol{\theta}} \equiv e^{i \boldsymbol{\theta} \cdot \boldsymbol{E}} S O(10)_{\mathrm{GFM}} e^{-i \boldsymbol{\theta} \cdot \boldsymbol{E}}
$$


contains $S U(5)_{\mathrm{GG}}$ as its subgroup. Thus the VEV

$$
\left\{\begin{array} { l } 
{ \langle S \rangle = v _ { \phi } \operatorname { c o s } ( \theta / 2 ) } \\
{ \langle \nu ^ { c } \rangle = v _ { \phi } \operatorname { s i n } ( \theta / 2 ) }
\end{array} \leftrightarrow \quad \left\{\begin{array}{c}
\left\langle S_{\theta}=S \cos (\theta / 2)+\nu^{c} \sin (\theta / 2)\right\rangle=v_{\phi} \\
\left\langle\nu_{\theta}^{c}=\nu^{c} \cos (\theta / 2)-S \sin (\theta / 2)\right\rangle=0
\end{array}\right.\right.
$$

breaks $E_{6}$ down to a twisted $S O(10), H_{\phi}=S O(10)_{\boldsymbol{\theta}}(24)$ with $\boldsymbol{\theta}=(0, \theta, 0)$. As a matter of fact, however, there is no loss of generality at this stage even if we assume that the $H_{\phi}$ symmetry is $S O(10)_{\mathrm{GFM}}=S O(10)_{\boldsymbol{\theta}=\mathbf{0}}$ with $\boldsymbol{\theta}$ set equal to zero. This is because we have no reference frame at this stage and we are free to define those $S U(2)_{E}$-rotated fields $S_{\theta}$ and $\nu_{\theta}^{c}$ simply to be $S$ and $\nu^{c}$. We can thus call $S O(10)_{\boldsymbol{\theta}}$ simply $S O(10)_{\mathrm{GFM}}$. If we have another reference frame, such as another VEV than $\langle\phi\rangle$, then, this freedom of twisting $S O(10)$ becomes to have a physical meaning and we will actually use it below.

Next consider the D breaking (20) by the adjoint Higgs $\Sigma(\mathbf{7 8})$. In order to specify the $S U(4)_{C}$ and $U(1)_{A} \times U(1)_{B}$ in the breaking pattern $\mathrm{D}$, it is convenient to consider a maximal subgroup $S U(6)_{C} \times S U(2)_{L}$ in $E_{6}$, under which the fundamental 27 decomposes into

$$
(\overline{\mathbf{1 5}}, \mathbf{1})=\left(\begin{array}{cccc}
-\varepsilon_{i k j} D^{k} & -u_{i}^{c} & -d_{i}^{c} & -D_{i}^{c} \\
u_{j}^{c} & 0 & S & \nu^{c} \\
d_{j}^{c} & -S & 0 & e^{c} \\
D_{j}^{c} & -\nu^{c} & -e^{c} & 0
\end{array}\right), \quad(\mathbf{6}, \mathbf{2})=\left(\begin{array}{cc}
u^{i} & d^{i} \\
E^{c} & -N^{c} \\
N & E \\
\nu & e
\end{array}\right) .
$$

Here the fist three entries and the last three entries of the $\mathbf{6}$ of $S U(6)_{C}$ are the fundamental representations 3 of $S U(3)_{C}$ and $\mathbf{3}$ of $S U(3)_{R}$, respectively. The three components of $\mathbf{3}$ of $S U(3)_{R}$ are arranged in the order for later convenience. We define and name three $S U(2)$ subgroups of the $S U(3)_{R}$ as follows by identifying their doublets:

$$
S U(2)_{R}:\left(\begin{array}{cc}
E^{c} & -N^{c} \\
N & E
\end{array}\right), \quad S U(2)_{R^{\prime}}:\left(\begin{array}{cc}
E^{c} & -N^{c} \\
\nu & e
\end{array}\right), \quad S U(2)_{E}:\left(\begin{array}{cc}
N & E \\
\nu & e
\end{array}\right) .
$$

The $S U(4)_{C}$ in the D breaking (20) should be $S U(4)_{C, \perp E}$ orthogonal to the $S U(2)_{E}$, whose fundamental representation 4 is given by the first four entries in the $S U(6)$ representation (26). The reason is as follows.

The true NG multiplets for the breaking $E_{6} \rightarrow G_{S}$ are given in Eq. (13)

$$
2\left(\hat{Q}_{1 / 6}+\hat{T}_{2 / 3}+S_{1}+S_{-1}\right)+\hat{Q}_{-5 / 6}+\left(\hat{T}_{-1 / 3}+\hat{D}_{1 / 2}\right)+4 S_{0}
$$

In addition to these we expect in this $(A, D)$ breaking scenario that there appear the following PsNG multiplets:

$$
(\mathbf{1 0}+\overline{\mathbf{1 0}})+\hat{D}_{1 / 2}+x S_{0}=\left(\hat{Q}_{1 / 6}+\hat{T}_{2 / 3}+S_{1}+S_{-1}\right)+\hat{D}_{1 / 2}+x S_{0}
$$


where the number $x$ of $G_{S}$-singlets $S_{0}$ can be arbitrary. On the other hand, the NG multiplets coming from the $\phi$-sector in which $E_{6} \rightarrow S O(10)_{\mathrm{GFM}}$ occurs are given in Eq. (11):

$$
\left(\hat{Q}_{1 / 6}+\hat{T}_{2 / 3}+S_{1}+S_{-1}\right)+\left(\hat{T}_{-1 / 3}+\hat{D}_{1 / 2}\right)+3 S_{0}
$$

Therefore the NG multiplets appearing from the $\Sigma$-sector should be

$$
2\left(\hat{Q}_{1 / 6}+\hat{T}_{2 / 3}+S_{1}+S_{-1}\right)+\hat{Q}_{-5 / 6}+\hat{D}_{1 / 2}+(1+x) S_{0}
$$

Note that the breaking in the $\Sigma$-sector is $E_{6} \rightarrow S U(4)_{C} \times S U(2)_{L} \times U(1)_{A} \times U(1)_{B}$ while the eventual breaking accompanied by the true NG multiplets is $E_{6} \rightarrow G_{S}=S U(3)_{C} \times$ $S U(2)_{L} \times U(1)_{Y}$. So the difference between (31) and (28),

$$
\hat{T}_{-1 / 3}+(3-x) S_{0}
$$

must correspond to the NG multiplets associated with the breaking $S U(4)_{C} \times U(1)_{A} \times$ $U(1)_{B} \rightarrow S U(3)_{C} \times U(1)_{Y}$. For the latter breaking we generally have $\hat{T}_{Y}+2 S_{0}$ as $\mathrm{NG}$ multiplets (so that $x$ is fixed to be 1). In order for this color triplet $\hat{T}_{Y}$ for the breaking $S U(4)_{C} \rightarrow S U(3)_{C}$ to carry the desired hypercharge $Y=-1 / 3$, the difference of $Y$ quantum number of the first three color triplet components from that of the fourth component of $S U(4)_{C} 4$ should be $-1 / 3$. Noting the hypercharge quantum numbers $Y\left(\left(u^{i}, d^{i}\right)\right)=1 / 6$, $Y\left(\left(E^{c},-N^{c}\right)\right)=+1 / 2, Y((N, E))=-1 / 2$ and $Y((\nu, e))=-1 / 2$, we see that the only possibility for $S U(4)_{C}$ is $S U(4)_{C, \perp E}$ for which the 4 is given by

$$
(\mathbf{6}, \mathbf{2})=\left(\begin{array}{cc}
u^{i} & d^{i} \\
E^{c} & -N^{c}
\end{array}\right) .
$$

Indeed then the generator which converts the fourth entry $E^{c}$ to $u$-quark $u^{i}$ is $S U(3)_{C}$ color triplet 3 and carries hypercharge $Y\left(u^{i}\right)-Y\left(E^{c}\right)=1 / 6-1 / 2=-1 / 3$ as required.

Now let us identify the VEV of $\Sigma(\mathbf{7 8})$ which realizes such D breaking $E_{6} \rightarrow S U(4)_{C, \perp E} \times$ $S U(2)_{L} \times U(1)_{A} \times U(1)_{B}$. As we have seen in Eq. (14), the adjoint $\Sigma(\mathbf{7 8})$ is decomposed under $S U(6)_{C} \times S U(2)_{L}$ as $\mathbf{7 8}=(\mathbf{1}, \mathbf{3})+(\mathbf{3 5}, \mathbf{1})+(\mathbf{2 0 , 2})$, the $\mathrm{VEV}\langle\Sigma\rangle$ realizing such a breaking is developed in the $S U(6)_{C}$ adjoint component $(\mathbf{3 5}, \mathbf{1})$ :

$$
\langle\Sigma(\mathbf{3 5}, \mathbf{1})\rangle=\left(\begin{array}{ccc}
a \mathbf{1}_{4} & 0 & 0 \\
0 & b & 0 \\
0 & 0 & c
\end{array}\right) \quad(4 a+b+c=0)
$$

Here this $6 \times 6$ matrix is written on the same basis as in Eq. (26) so that the bottom right $2 \times 2$ submatrix corresponds to $S U(2)_{E} \times U(1)$. Note that we have used $S U(6)_{C}$ rotations to bring the generic VEV of hermitian $6 \times 6$ matrix $\Sigma(\mathbf{3 5}, \mathbf{1})$ into the above diagonal form; in 
particular, an $S U(2)_{E}$ rotation is used to make the bottom right $2 \times 2$ submatrix diagonal. This means that the previous $\phi$-sector unbroken subgroup $H_{\phi}$ no longer remains to be the $S O(10)_{\text {GFM }}$ with $\theta=0$ in this basis but becomes $S O(10)_{\boldsymbol{\theta}}$ with $\theta \neq 0$. For $\theta \neq 0$ to have a physical meaning, the $S U(2)_{E}$ must be broken by $b \neq a$ as we assume here. Then two unbroken $U(1)$ charges, called $U(1)_{A}$ and $U(1)_{B}$ in the above, are given in this basis by

$$
U(1)_{A}: A \equiv\left(\begin{array}{ccc}
\mathbf{1}_{4} & 0 & 0 \\
0 & -2 & 0 \\
0 & 0 & -2
\end{array}\right), \quad U(1)_{B}: B \equiv\left(\begin{array}{ccc}
\mathbf{0}_{4} & 0 & 0 \\
0 & 1 & 0 \\
0 & 0 & -1
\end{array}\right)=E_{3} .
$$

The latter charge $B$ is chosen to be the third component $E_{3}$ of $S U(2)_{E}$.

It should be emphasized that $\theta$ must not be zero. Otherwise, the intersection $H_{\phi} \cap H_{\Sigma}$ would contain an extraneous $U(1)$ other than the standard theory gauge symmetry $G_{S}$. Indeed, if $H_{\phi}=S O(10)_{\mathrm{GFM}}$, its five Cartan generators are all diagonal in the particle basis which we have defined in Eq. (21), while $H_{\Sigma}=S U(4)_{C, \perp E} \times S U(2)_{L} \times U(1)_{A} \times U(1)_{B}$ is rank 6 and contains all the Cartan generators in $E_{6}$, which are also diagonal on the same basis. Therefore the $U(1)_{V}$ contained in $S O(10)_{\mathrm{GFM}} \supset S U(5)_{\mathrm{GG}} \times U(1)_{V}$ can be necessarily written as a linear combination of the six Cartan generators in $H_{\Sigma}$ and hence remains as an unbroken symmetry contained in the intersection $H_{\phi} \cap H_{\Sigma}$ in contradiction to the assumption. If $\theta \neq 0$, on the other hand, the directions of Cartan generators in $H_{\phi}$ and $H_{\Sigma}$ are twisted and no such $U(1)$ remains. [This can be seen by looking at, e.g., $e^{-i \theta E_{2}} E_{3} e^{i \theta E_{2}}=E_{3} \cos \theta+E_{1} \sin \theta$.]

Finally let us confirm the quantum numbers including the hypercharge of the NG multiplets which actually appear in this D breaking $E_{6} \rightarrow S U(4)_{C, \perp E} \times S U(2)_{L} \times U(1)_{A} \times U(1)_{B}$ realized by the $\Sigma$-VEV (34). Noting the hypercharge $Y$ is given by

$$
Y=\left(\begin{array}{ccc}
(1 / 6) \mathbf{1}_{3} & 0 & 0 \\
0 & 1 / 2 & 0 \\
0 & 0 & (-1 / 2) \mathbf{1}_{2}
\end{array}\right)
$$

on the fundamental representation 6 of $S U(6)_{C}$ in the basis $(26)$, we can find the $S U(3)_{C} \times$ $U(1)_{Y}$ quantum numbers of the $\mathbf{2 0}=$ 日 by inspecting Eq. (15): ${ }^{*}$

$$
\begin{aligned}
\mathbf{2 0} & =(\mathbf{4}, \mathbf{1})+(\overline{\mathbf{4}}, \mathbf{1})+(\mathbf{6}, \mathbf{2}) \quad \text { under } S U(4)_{C, \perp E} \times S U(2)_{E} \\
& =\left(\mathbf{3}_{-5 / 6}+\mathbf{1}_{-1 / 2}, \mathbf{1}\right)+\left(\overline{\mathbf{3}}_{5 / 6}+\mathbf{1}_{1 / 2}, \mathbf{1}\right)+\left(\mathbf{3}_{1 / 6}+\overline{\mathbf{3}}_{-1 / 6}, \mathbf{2}\right)
\end{aligned}
$$

so that the $G_{S}$ quantum numbers of $(\mathbf{2 0}, \mathbf{2})$, which appears for the breaking $E_{6} \rightarrow S U(6)_{C} \times$ $S U(2)_{L}$, are given by

$$
(\mathbf{2 0}, \mathbf{2})=\hat{Q}_{-5 / 6}+\hat{D}_{1 / 2}+2 \hat{Q}_{1 / 6}
$$

*) For instance, the hypercharge $Y=-5 / 6$ for $\left(3_{-5 / 6}, \mathbf{1}\right)$ can be found as follows. It corresponds to $\frac{\alpha}{\cdot}$ with color index $\alpha=1,2,3$ and $\cdot=5$ or 6 , hence carrying the hypercharge $(1 / 6)+(-1 / 2)+(-1 / 2)=-5 / 6$. 
When $S U(6)_{C} \times S U(2)_{L}$ is further broken to $S U(4)_{C, \perp E} \times S U(2)_{L} \times U(1)_{A} \times U(1)_{B}$, the appearing NG multiplets are

$$
\begin{aligned}
& (\mathbf{4}, \overline{\mathbf{2}})+(\overline{\mathbf{4}}, \mathbf{2})+2 S_{0} \quad \text { under } S U(4)_{C, \perp E} \times S U(2)_{E} \\
& \quad=(\mathbf{3}, \overline{\mathbf{2}})_{2 / 3}+(\mathbf{1}, \overline{\mathbf{2}})_{1}+(\overline{\mathbf{3}}, \mathbf{2})_{-2 / 3}+(\mathbf{1}, \mathbf{2})_{-1}+2 S_{0} \\
& \quad=2 \times\left(\hat{T}_{2 / 3}+S_{1}+S_{-1}\right)+2 S_{0}
\end{aligned}
$$

where $2 S_{0}$ comes from the breaking $S U(2)_{E} \rightarrow U(1)_{E_{3}}$. We thus see that the resultant NG multiplets (38) plus (39) indeed realizes the expected one in Eq. (31).

\section{Proton Decay:}

The important prediction of the present idea of Higgs doublets as PsNG multiplets is that there necessarily appear additional PsNG multiplets $\mathbf{1 0}_{H}+\overline{\mathbf{1 0}}_{H}$ of $S U(5)_{\mathrm{GG}}$ which we expect will get masses $M_{\mathbf{1 0}}$ around $O(1) \mathrm{TeV}$ after SUSY is broken. Aside from the direct observation of them, their effect may be seen through proton decay. Let us evaluate the order of the proton decay caused by their effect.

We expect generically the presence of the following dimension 4 and 5 operators in the low energy effective superpotential: in terms of the $S U(5)$ language,

$$
\begin{aligned}
& W_{4}=f_{4}^{i j} \overline{\mathbf{5}}_{i} \overline{\mathbf{5}}_{j} \mathbf{1 0}_{H} \supset f_{4}^{i j}\left[\epsilon^{\alpha \beta \gamma} d_{i \alpha}^{c} d_{j \beta}^{c} u_{H \gamma}^{c}+d_{i \alpha}^{c}\left(e_{j} u_{H}^{\alpha}-\nu_{j} d_{H}^{\alpha}\right)\right] \\
& W_{5}=\frac{f_{5}^{i j}}{M_{\mathrm{pl}}} \mathbf{1 0}_{i} \overline{\mathbf{5}}_{j} \mathbf{5}_{H} \overline{\mathbf{1 0}}_{H} \supset f_{5}^{i j}\left[\epsilon^{\alpha \beta \gamma} u_{i \alpha}^{c} d_{j \beta}^{c}+\left(u_{i}^{\gamma} e_{j}-d_{i}^{\gamma} \nu_{j}\right)\right]\left\langle H_{u}\right\rangle \bar{d}_{H \gamma},
\end{aligned}
$$

where $\mathbf{1 0}_{i}$ and $\overline{\mathbf{5}}_{i}(i=1,2,3)$ denotes three generations of matters, $\mathbf{1 0}_{H}$ and $\overline{\mathbf{1 0}}_{H}$ are our new light Higgs, and $\mathbf{5}_{H}$ is the usual Higgs $H_{u}$ in which the color triplet part is in fact missing. If the colored components in $\mathbf{1 0}_{H}$ and in $\overline{\mathbf{1 0}}_{H}$ are connected by propagator $\left\langle\mathbf{1 0}_{H} \overline{\mathbf{1 0}}_{H}\right\rangle$ and the usual Higgs doublet $\mathbf{5}_{H}$ is replaced by the $\operatorname{VEV}\left\langle H_{u}\right\rangle$, then we have an effective superpotential which breaks baryon number:

$$
W_{6}=\frac{f_{\mathrm{eff}}^{i j k l}}{M_{\mathrm{pl}}} \epsilon^{\alpha \beta \gamma} u_{i \alpha}^{c} d_{j \beta}^{c} \times d_{k \gamma}^{c} \nu_{l}, \quad f_{\mathrm{eff}}^{i j k l}=f_{5}^{i j} f_{4}^{k l} \frac{\left\langle H_{u}\right\rangle}{M_{\mathbf{1 0}}}
$$

Note that if the Higgs VEV $\left\langle H_{u}\right\rangle$ is replaced by the Higgs superfield, then this term gives an dimension 6 operator but the suppression is not by the square of Planck mass $M_{\mathrm{pl}}$ but by a single power of $M_{\mathrm{pl}}$. Another mass scale $M_{\mathbf{1 0}}$ comes from the propagator of $\mathbf{1 0}_{H}$ Higgs

which is light and does not give any significant suppression; $\left\langle H_{u}\right\rangle / M_{10} \sim 1-10^{-1}$. So this operator is potentially dangerous so that the proton decay by this operator should be suppressed by the smallness of the coupling constant.

Similarly to the analysis of the generic dimension 5 operators as performed by Kakizaki and Yamaguchi, ${ }^{14}$ ) we can think that the coupling constants $f_{4}^{i j}$ and $f_{5}^{i j}$ obey a Froggatt- 
Nielsen ${ }^{15)}$ suppression mechanism similar to the usual Higgs Yukawa coupling constant responsible for the fermion masses. Then, using letters $q_{i}, l_{i}, u_{i}^{c}, d_{i}^{c}$ and $h_{u}, h_{d}$ to denote the Froggatt-Nielsen $U(1)$ charges of $i$-th generation quarks and leptons and the up- and downtype Higgs doublets $H_{u}$ and $H_{d}$,

$$
\begin{aligned}
f_{5}^{i j} f_{4}^{k l} & =f_{4} f_{5} \lambda^{u_{i}^{c}+d_{j}^{c}+d_{k}^{c}+l_{l}+h_{u}}, \quad f_{4,5} \sim O(1) \\
& =\left(f_{4} f_{5}\right) y_{d}^{i j} \lambda^{\left(u_{i}^{c}-q_{i}\right)-h_{d}} y_{d}^{l k} \lambda^{\left(l_{l}-q_{l}\right)-h_{d}+h_{u}}
\end{aligned}
$$

where $\lambda \sim \sin \theta_{C}=0.22$ and $y_{d}^{i j}=\lambda^{q_{i}+u_{j}^{c}+h_{d}}$ is the $i j$ matrix element of the down-type quark yukawa coupling. Then the largest operator is

$$
W_{6}=\frac{f_{\mathrm{eff}}^{1123}}{M_{\mathrm{pl}}} u_{R} d_{R} s_{R} \nu_{\tau},
$$

so that the main decay mode is $p \rightarrow K^{+} \bar{\nu}_{\tau}$. The bound for the proton lifetime $\tau_{\text {proton }}>$ $2 \times 10^{33} \mathrm{yr}$ gives a constraint

$$
\left|f_{\mathrm{eff}}^{i j k l}\right| \lesssim\left(10^{-7} \sim \lambda^{11}\right) \times\left(\frac{M_{\mathrm{pl}}}{10^{19} \mathrm{GeV}}\right)
$$

We have for the main decay mode

$$
f_{\mathrm{eff}}^{1123} \simeq\left(f_{4} f_{5}\right) \frac{\left\langle H_{u}\right\rangle}{M_{\mathbf{1 0}}} \times\left(y_{d}^{11} \lambda^{\left(u_{1}^{c}-q_{1}\right)-h_{d}} y_{d}^{32} \lambda^{\left(l_{3}-q_{3}\right)-h_{d}+h_{u}}=y_{d}^{11} y_{d}^{32} \lambda^{p+2 h_{u}-3 h_{d}}\right)
$$

where use has been made of the 'GUT-inspired' relations $q_{i}=u_{i}^{c}$ and $l_{i}=d_{i}^{c}$ by Kakizaki and Yamaguchi and of the definition of $p$ :

$$
y_{b} / y_{t}=\lambda^{d_{3}^{c}+h_{d}-u_{3}^{c}-h_{u}} \equiv \lambda^{p} \quad \rightarrow \quad l_{3}-q_{3}=p+h_{u}-h_{d}
$$

If we use $p=2$ corresponding $\tan \beta \simeq 3$, and semi-empirical relations $y_{d}^{11}=\lambda^{5} y_{b}, y_{d}^{32}=$ $y_{d}^{33} \equiv y_{b}$ and $y_{t} \sim 1$, we have

$$
f_{\mathrm{eff}}^{1123} \simeq\left(f_{4} f_{5}\right) \frac{\left\langle H_{u}\right\rangle}{M_{10}} \times \lambda^{11+2 h_{u}-3 h_{d}}
$$

Therefore, since it is natural to expect that the factors $\left(f_{4} f_{5}\right) \frac{\left\langle H_{u}\right\rangle}{M_{10}}$ and $\lambda^{2 h_{u}-3 h_{d}}$ are of order 1 , we could see the proton decay in near future.

\section{Yukawa couplings:}

The particular property of our Higgs doublets as PsNG multiplets is their representations under $S O(10) \subset E_{6}$. As is seen from the discussion above, in particular Eq. (11), the downtype Higgs $H_{d}$ is contained in $(\mathbf{1 6}, \overline{\mathbf{5}})$ in $\phi(\mathbf{2 7})$ and up-type Higgs $H_{u}$ in $(\overline{\mathbf{1 6}}, \mathbf{5})$ in $\phi(\overline{\mathbf{2 7}})$, where the two numbers in the brackets denote representations under $S O(10)$ and $S U(5)$. This 
is in sharp contrast with the usual GUTs in which $H_{u}$ and $H_{d}$ are assigned to be $(\mathbf{1 0}, \mathbf{5})$ and $(\mathbf{1 0}, \overline{\mathbf{5}})$. This property leads to some peculiarities in obtaining fermion mass terms in this model. The down-type quark mass terms come from the usual trilinear terms but they actually exist only when the down-type quarks contain ' $S U(2)_{E}$ twisted components' $\Psi(\mathbf{1 0}, \overline{5})$ :

$$
\Psi_{i}(\mathbf{2 7}) \Psi_{j}(\mathbf{2 7}) \phi(\mathbf{2 7}) \rightarrow \Psi_{i}(\mathbf{1 6}, \mathbf{1 0}) \Psi_{j}(\mathbf{1 0}, \overline{\mathbf{5}}) \phi(\mathbf{1 6}, \overline{\mathbf{5}}) .
$$

Since $\phi(\mathbf{2 7})$ does not contain the up-type Higgs $H_{u} \subset \mathbf{5}$, these trilinear terms do not contain up-type quark masses at all. Up-type quark mass terms come from dimension 5 operators:

$$
\Psi_{i}(\mathbf{2 7}) \Psi_{j}(\mathbf{2 7}) \phi(\overline{\mathbf{2 7}}) \phi(\overline{\mathbf{2 7}}) \rightarrow \Psi_{i}(\mathbf{1 6}, \mathbf{1 0}) \Psi_{j}(\mathbf{1 6}, \mathbf{1 0}) \phi(\overline{\mathbf{1 6}}, \overline{\mathbf{5}})\langle\phi(\overline{\mathbf{1 6}}, \overline{\mathbf{1}})\rangle .
$$

Note that the $\operatorname{VEV}\langle\phi(\overline{\mathbf{1 6}}, \overline{\mathbf{1}})\rangle$ is non-vanishing only when the $S U(2)_{E}$ rotation (24) in the Higgs sector exists, $\theta \neq 0$. The induced top Yukawa copling is thus not of dimension 4 coupling but comes from a higher dimensional operator. The resultant Yukawa couplings are thus accompanied with $\langle\phi(\overline{\mathbf{1 6}}, \overline{\mathbf{1}})\rangle / M_{P}$. This eventually suppress the top Yukawa coupling by the power $\lambda$ or so. Note that the bottom Yukawa coupling can in principle be dimension 4. However we expect the so-called family twisting structure ${ }^{12), 16), 13)}$ and so the bottom Yukawa couplings may be accompanied by some Froggatt-Nielsen factor, so that the ratio of Yukawa couplings of the top and the bottom quarks can become smaller. Also note that in our scenario the unified gauge coupling is larger than the usual case and it may be possible to get a reasonable top quark mass as an quasi infrared fixed point; the running Yukawa coupling approaches to the order of color gauge coupling faster than in the usual case *)

We conclude this note by adding some comments.

The PsNG Higgs approach based on the model with $G=S U(6) \times S U(2)_{R}$ gauge symmetry instead of $E_{6}$ may also be interesting, ${ }^{17)}$ in which the breaking pattern is given by

$$
\begin{aligned}
& {\left[S U(6) \times S U(2)_{R}\right]_{\phi} \longrightarrow S U(5)_{\mathrm{GG}},} \\
& {\left[S U(6) \times S U(2)_{R}\right]_{\Sigma} \longrightarrow S U(4)_{C} \times S U(2)_{L} \times S U(2)_{R} \times U(1)}
\end{aligned}
$$

and there appears no extra PsNG multiplet than the desired Higgs doublets. This breaking pattern can be realized by the $\phi$ and $\Sigma$ Higgs sectors which consist of $\phi(\overline{\mathbf{6}}, \mathbf{2})$ and $\Sigma(\mathbf{1 5}, \mathbf{1})$ in addition to their conjugates, respectively. Note that $\Sigma(\mathbf{1 5}, \mathbf{1})$ contains no $S U(5)$ singlet component but has an $S U(4)$ singlet. So it can naturally breaks $S U(6)$ down to $S U(4)$ instead $S U(5)$. Moreover these Higgs fields $\phi(\overline{\mathbf{6}}, \mathbf{2})$ and $\Sigma(\mathbf{1 5}, \mathbf{1})$ can be combined into a

*) The same problem already happens in the $S U(6)$ case considered by Dvali and Pomarol. ${ }^{10)}$ They introduced an additional fermion fields 20, and top quark is represented as a mixture state of $\mathbf{1 5}$ with $\mathbf{2 0 .}$ This provides a dimension 4 top Yukawa coupling. 
fundamental representation 27 of $E_{6}$ representation. So if $E_{6}$ is broken by some mechanism, for example by Hosotani mechanism, it may be possible to make a realistic scenario by using only the fundamental representation Higgs.

The notion of PsNG bosons were first investigated intensively by S. Weinberg ${ }^{18)}$ in the context of dynamical symmetry breaking. The essential difference between his PsNG and the present one is the existence of SUSY. In the non-SUSY case the mass of PsNG is generated via residual gauge interaction which breaks the tree level symmetry and the order is estimated to be $m^{2}=g^{2} \Lambda^{2}$, with $\Lambda$ being a characteristic scale of the interaction responsible for the spontaneous breaking. In SUSY case, on the other hand, the masses of PsNG fields are protected until the SUSY breaking occurs. This ensures masses of PsNG very light of the order $\sim g M_{\mathrm{SUSY}}$.

We would like to stress that $E_{6}{ }^{19)}$ model has many advantages. Especially after the recent neutrino oscillation observations confirmed the remarkable fact of the neutrino large mixings, $E_{6}$ model became more attractive because we anyhow need some non-parallel (twisting) family structure in order to reproduce those large mixings. ${ }^{20)} E_{6}$ provides us with the most natural scenario for realizing this twisting family structure. ${ }^{13), 16)}$ We have seen in this paper that this twisting structure is also required in the symmetry breaking pattern to assure the intersection $H_{\phi} \cap H_{\Sigma}$ reduces to the standard theory gauge group $G_{S}$.

\section{Acknowledgments}

We would like to thank to H. Haba, N. Maekawa, S.. Yamashita, and M. Kakizaki for stimulating discussions. M. B. and T. K. are supported in part by the Grant-in-Aid for Scientific Research Nos. 12640295 and 13640279, respectively, from Japan Society for the Promotion of Science, and Grants-in-Aid for Scientific Research on Priority Area A "Neutrinos" (Y. Suzuki) Nos. 12047225 and 12047214, respectively, from the Ministry of Education, Science, Sports and Culture, Japan. Also we are stimulated by the fruitful and instructive discussions during the Summer Institute 2001 and 2002 held at Fuji-Yoshida.

\section{References}

1) E. Witten, Phys. Lett. B105 (1981), 267; Nucl. Phys. B258 (1985), 75.

A. Masiero, D. V. Nanopoulos, K. Tamvakis and T. Yanagida, Phys. Lett. 115 (1982), 380; Phys. Lett. B344 (1995), 211.

Y. Kawamura, Prog. Theor. Phys. 105 (2001), 999.

2) N. Maekawa and T. Yamashita, Prog. Theor. Phys. 107 (2002) 1201 [arXiv:hep- 
$\mathrm{ph} / 0202050]$.

N. Maekawa, Prog. Theor. Phys. 106 (2001) 401 [arXiv:hep-ph/0104200].

3) S. Dimopoulos and F. Wilczek, NSF-ITP-82-07.

M. Srednicki, Nucl. Phys. B202 (1982), 327.

4) S. M. Barr and S. Raby, Phys. Rev. Lett. 79 (1997) 4748 [arXiv:hep-ph/9705366].

5) K. Inoue, A. Kakuto and H. Takano, Prog. Theor. Phys. 75 (1986), 664.

6) A. A. Anselm and A. A. Johansen, Phys. Lett. B 200 (1988) 331.

7) Z. G. Berezhiani and G. R. Dvali, Bull. Lebedev Phys. Inst. 5 (1989) 55 [Kratk. Soobshch. Fiz. 5 (1989) 42].

8) R. Barbieri, G. R. Dvali and M. Moretti, Phys. Lett. B 312 (1993) 137.

9) Z. Berezhiani, C. Csaki and L. Randall, Nucl. Phys. B 444 (1995) 61 [arXiv:hep$\mathrm{ph} / 9501336]$.

10) G. R. Dvali and S. Pokorski, Phys. Rev. Lett. 78 (1997) 807 [arXiv:hep-ph/9610431]; Phys. Lett. B 379 (1996) 126 [arXiv:hep-ph/9601358].

11) M. Bando, T. Kuramoto, T. Maskawa and S. Uehara, Phys. Lett. B 138 (1984) 94; Prog. Theor. Phys. 72 (1984) 313; Prog. Theor. Phys. 72 (1984) 1207.

12) M. Bando and T. Kugo, Prog. Theor. Phys. 101 (1999) 1313 [arXiv:hep-ph/9902204].

13) M. Bando, T. Kugo and K. Yoshioka, Prog. Theor. Phys. 104 (2000) 211 [arXiv:hepph/0003220]; Phys. Lett. B 483 (2000) 163 [arXiv:hep-ph/0003231].

M. Bando, "Twisted family structure and neutrino large mixing," arXiv:hep$\mathrm{ph} / 0005229$.

14) M. Kakizaki and M. Yamaguchi, JHEP 0206 (2002) 032 [arXiv:hep-ph/0203192]; arXiv:hep-ph/0110266.

15) C. D. Froggatt and H. B. Nielsen, Nucl. Phys. B147 (1979), 277.

16) M. Bando and N. Maekawa, Prog. Theor. Phys. 106 (2001) 1255 [arXiv:hep$\mathrm{ph} / 0109018]$.

17) N. Haba, C. Hattori, M. Matsuda and T. Matsuoka, Prog. Theor. Phys. 96 (1996), 1249; M. Matsuda and T. Matsuoka, Phys. Lett. B487 (2000), 104.

18) S. Weinberg, Phys. Rev. 13 (1976), 974.

19) F. Gürsey, P. Ramond and P. Sikivie, Phys. Lett. 60B (1976), 177.

Y. Achiman and B. Stech, Phys. Lett. 77B (1978), 389.

P. Ramond, Sanibel Symp. (Florida, 1979) CALT-68-709.

20) T. Yanagida, Talk given at 18th International Conference on Neutrino Physics and Astrophysics (NEUTRINO 98), Takayama, Japan, 4-9 Jun 1998; hep-ph/9809307. 